\title{
Transnationale Zusammenarbeit zur Raumentwicklung - Herausbildung, Stand und Perspektiven bis 2013
}

\section{Einleitung}

Transnationale Zusammenarbeit zur Raumentwicklung ist seit Mitte der 1990er Jahre zu einem festen Bestandteil der EU-Strukturpolitik geworden, zunächst als Gemeinschaftsinitiative und künftig als Teil einer von drei Grundrichtungen der Strukturförderung. Mit der transnationalen Zusammenarbeit fördert die EU im Sinne einer polyzentrischen Raumentwicklung einerseits die Entwicklung einer Anzahl staatenübergreifender Kooperationsräume und damit mehrerer „Kraftzentren“ in Europa. Andererseits wird auch das Zusammenwachsen der EU „von unten“ durch konkrete transnationale Projekte vorangebracht.

Die Inhalte und Qualitätsmerkmale der transnationalen Zusammenarbeit für den Zeitraum 2007-2013 sind einerseits Ergebnis der inneren Dynamik transnationaler Förderprogramme seit der ersten Pilotphase von 1996 bis 1999; so analysierten die EU-Mitgliedstaaten und die Europäische Kommission die Ergebnisse transnationaler Programme und Projekte und zogen Schlussfolgerungen für ihre Weiterentwicklung. Andererseits sind die neuen Programme auch Ergebnis einer grundsätzlichen Neuorientierung der Wirtschafts- und Umweltpolitik im Ergebnis der Gipfeltreffen der Regierungschefs von Lissabon und Göteborg.

Insgesamt haben transnationale Programme die Herausbildung komplexer und integrierter Entwicklungsansätze für europäische Großräume gefördert, die über die traditionelle Regionalpolitik weit hinausgehen. Die Projekte haben sich dabei von staatenübergreifenden Studien zu strategischen Raumentwicklungsprojekten entwickelt, die breit anwendbare Modelllösungen schaffen und künftige Investitionen vorbereiten helfen.

\section{Transnationale Zusammenarbeit und Strukturpolitik}

Nicht erst seit Einführung des Begriffs des territorialen Zusammenhalts sind staatenübergreifende Herangehensweisen Bestandteil europäischer Strukturpolitik. Nach Artikel 158 des Vertrags zur Gründung der Euro- päischen Gemeinschaften (EGV) ist die Weiterentwicklung und Förderung der Politik zur Stärkung des wirtschaftlichen und sozialen Zusammenhalts eines der zentralen Ziele der Gemeinschaft. Die Strukturpolitik mit ihren räumlichen Orientierungen nimmt eine bedeutende Stellung bei der Umsetzung dieses Ziels ein. So sollen die Unterschiede im Entwicklungsstand der verschiedenen Regionen verringert und der Rückstand der am stärksten benachteiligten Gebiete oder Inseln unter Einschluss der ländlichen Gebiete reduziert werden.

Im Zuge der EU-Erweiterung vergrößerten sich auch die internen Entwicklungsunterschiede zwischen den Regionen. Zeichneten sich die sechs Gründerstaaten der Europäischen Gemeinschaft noch durch eine relativ homogene Wirtschaftsstruktur aus (mit Ausnahme des italienischen Mezzogiorno), so wurden die regionalen Disparitäten mit jeder Erweiterung der Gemeinschaft offensichtlicher. Die europäische Strukturpolitik entwickelte sich zum zentralen Instrument, um diese Unterschiede abzumildern und die Wettbewerbsfähigkeit der EU nachhaltig zu sichern und zu verbessern. Dabei lag der Fokus der Gemeinschaftspolitik zunächst vorwiegend auf wirtschaftspolitischen Maßnahmen, nicht jedoch auf dem Raum bzw. einer Raumentwicklungspolitik mit einer ganzheitlichen Herangehensweise.

\section{Raumentwicklung in der europäischen Diskussion}

Dies änderte sich zum Ende der 1980er und Beginn der 1990er Jahre. Bereits der Europarat hatte Jahre zuvor die Bedeutung von zwei Faktoren für den Erfolg der europäischen Integration herausgestellt, nämlich der aktiven Beteiligung und Einbindung der europäischen Bürger in den Integrationsprozess und der grenzüberschreitenden Zusammenarbeit der Regionen und Kommunen insbesondere an den EU-Binnengrenzen.

Die Erarbeitung und Verabschiedung der Europäischen Raumordnungscharta im Jahr 1983 durch den Europarat kann als Pionierleistung für die Befassung mit einer europäischen Raumentwicklungspolitik bezeichnet werden. Dieses Dokument spannt erstmals einen 
Rahmen für wichtige Orientierungspunkte einer Politik der räumlichen Ordnung und für Maßnahmen zur Verbesserung der Lebensqualität auf gesamteuropäischer Ebene. Die Charta stellt die Bedeutung der Raumentwicklung für die Entwicklung der Gesellschaft in Europa heraus und sieht in einer entsprechenden Politik einen wesentlichen Beitrag zur Entstehung einer europäischen Identität. Mit den vier Grundsätzen einer europäischen Raumentwicklungspolitik

(1) ausgewogene soziale und wirtschaftliche Entwicklung der Regionen,

(2) Erhöhung der Lebensqualität der Bevölkerung,

(3) verantwortungsvolle Behandlung der natürlichen Ressourcen und der Schutz der Umwelt sowie

(4) planmäßige und rationelle/haushälterische Nutzung des Raums ${ }^{1}$

bezieht sich die Charta bereits eindeutig auf die Zielsetzungen einer nachhaltigen Entwicklung des europäischen Raums und auf die Stärkung des wirtschaftlichen und sozialen Zusammenhalts. Die Charta stellt somit als erstes Dokument die Verbindung zwischen europäischer Strukturpolitik und einer ganzheitlichen Raumentwicklung in Europa her und liefert einen wichtigen Ansatzpunkt für die später stärkere Berücksichtigung der räumlichen Entwicklung und Zusammenarbeit in der EU-Strukturpolitik.

Innerhalb der Europäischen Gemeinschaft wurde ab Ende der 1980er Jahre - wegen des zunehmenden Einflusses europäischer Fachpolitiken auf die räumliche Entwicklung der Mitgliedstaaten sowie ihrer Städte und Regionen - die Notwendigkeit einer Raumordnungsbzw. Raumentwicklungspolitik auf Gemeinschaftsebene diskutiert. Die EG-Verträge von Paris und Rom oder der EU-Vertrag von Maastricht sehen kein Mandat der Gemeinschaft für Fragen der Raumordnung vor. Allerdings wurde im Zuge des Maastrichter Vertrags ein stärker raumwirksames Tätigwerden der Gemeinschaft auf einer Reihe von Politikfeldern ermöglicht.

Diese Entwicklung, dass die Europäische Kommission ohne eine eigenständige raumordnerische Kompetenz faktisch Raumentwicklungspolitik betreiben konnte, verstärkte - auch schon im Vorfeld des Ratifizierungsprozesses - den Ruf nach einer europäischen Raumordnungspolitik, wobei die Frage der Kompetenzen und Aufgabenverteilung zwischen der EU und den Mitgliedstaaten durchaus kontrovers diskutiert wurde. Die „intensive Diskussion um ein Für und Wider europäischer Raumordnungspolitik“2 löste gleichzeitig verschiedene raumentwicklungspolitische Initiativen der Mitgliedstaaten und der Kommission aus.
So wurde auf dem ersten, 1989 unter französischer Ratspräsidentschaft einberufenen informellen Treffen der Minister für Raumordnung und Regionalpolitik in Nantes die Idee geboren, eine gemeinsame Position und Perspektive zur europäischen Entwicklung zu erarbeiten. ${ }^{3}$ Die Europäische Kommission nahm dies zum Anlass, mit den 1991 bzw. 1994 vorgelegten Dokumenten „Europa 2000 " 4 und „Europa 2000+ ${ }^{\circ}$. wichtige Informationen und Grundlagen für politische Entscheidungen zur Raumentwicklung in Europa bereitzustellen. In einem interaktiven Prozess trieben dann die Mitgliedstaaten die Arbeiten an einem gemeinsamen Raumentwicklungskonzept für das Gebiet der EU voran. Der informelle Raumordnungsministerrat nahm 1994 in Leipzig ein Grundlagendokument hierzu an, in dem erstmals auch die Ausweisung „Europäischer Aktionsräume für eine integrierte räumliche Entwicklung" angeregt wurde. ${ }^{6}$ In diesen Räumen sollten moderne Verwaltungsstrukturen gestärkt, Leitbilder ausgearbeitet und umgesetzt und die notwendigen Koordinierungsmechanismen eingerichtet werden. ${ }^{7}$ Parallel dazu zeigt die Europäische Kommission mit dem Dokument „Europa 2000+ " für solche transnationalen Teilräume Europas alternative Entwicklungsszenarien auf. Darüber hinaus stellt sie in politischen Schlussfolgerungen die Entwicklung transnationaler Programme in Aussicht. ${ }^{8}$

\section{Auf dem Weg zu einem Raumentwicklungskonzept für die $E U$}

Die genannten Prozesse mündeten schließlich in das "Europäische Raumentwicklungskonzept“ (EUREK), das in mitgliedstaatlichem Zusammenwirken erarbeitet und 1999 in Potsdam als zentrales Leitliniendokument für eine nachhaltige räumliche Entwicklung in der EU von den zuständigen Ministern angenommen wurde. Damit hatten sich die Mitgliedstaaten und die Europäische Kommission auf gemeinsame räumliche Ziele für die zukünftige Entwicklung des EU-Territoriums verständigt. ${ }^{9}$

Herausgestellt werden in dem Dokument das Fortschreiten des europäischen Integrationsprozesses und die Verringerung des trennenden Charakters von Binnengrenzen. So lassen sich angesichts der zunehmenden Verflechtung von Regionen und Städten innerhalb der Gemeinschaft immer weniger Herausforderungen aus einem rein nationalen Kontext heraus bewältigen. Vielmehr fordert die Lösung räumlicher Entwicklungsprobleme und damit die Verwirklichung des Ziels des wirtschaftlichen und sozialen Zusammenhalts eine verstärkte Kooperation unterschiedlicher Regierungs- und Verwaltungsebenen. 
Das EUREK bietet aber nicht nur einen Orientierungsrahmen für eine verbesserte Kooperation zwischen unterschiedlichen administrativen Ebenen, sondern betont auch, dass die Weiterentwicklung der europäischen Verträge zu einem immer stärkeren Einfluss der europäischen Fachpolitiken auf die räumliche Entwicklung der EU geführt hat. Diese räumlichen Auswirkungen ergänzen sich nicht zwangsläufig im Sinne einer stärker regional ausgewogenen Entwicklung. ${ }^{10}$ Davon ausgehend wurde der Blick über rein fachpolitische Maßnahmen hinaus auf die Gesamtsituation des europäischen Territoriums gerichtet. Diese übergreifende Betrachtung der Auswirkungen der Gemeinschaftspolitiken auf das Gebiet der EU stellt ein Novum dar.

\section{Transnationale Zusammenarbeit zur Raumentwicklung wird Gemeinschaftsinitiative}

Im Zuge der dargestellten Aktivitäten reifte die Erkenntnis, dass eine integrierte räumliche Entwicklungspolitik besser verwirklicht werden kann, wenn sie in die europäische Strukturpolitik integriert ist. Mit der 1996 aufgelegten Gemeinschaftsinitiative INTERREG IIC hat die Europäische Kommission bereits in der Erarbeitungsphase des EUREK ein Förderprogramm geschaffen, mit dem sie die transnationale Zusammenarbeit der Mitgliedstaaten und ihrer Städte und Regionen in größeren europäischen Räumen auf dem Gebiet der Raumordnung initiiert und unterstützt. In diesen Räumen erarbeiten die Mitgliedstaaten und ihre Städte und Regionen gemeinsame Entwicklungsvorstellungen, die sie in Projekten umsetzen. Zum ersten Mal wurden damit Mittel der europäischen Strukturpolitik für ein Programm eingesetzt, das sektorübergreifend agiert und eine integrierte Raumentwicklung auf dem Gebiet der EU unterstützt. Die Zusammenarbeit erfolgt im Kontext großer, staatenübergreifender Kooperationsräume. Die Initiative erreicht damit eine deutlich größere räumliche Wirksamkeit als die grenzüberschreitende Kooperation. Mit ihr werden komplexe Entwicklungsansätze auf transnationale Teilräume übertragen und Lösungen für aktuelle Herausforderungen der Raumentwicklung kooperativ erarbeitet.

Diese Förderung der transnationalen Zusammenarbeit durch die europäische Strukturpolitik leistet seit ihren Anfängen einen wichtigen Beitrag zum Abbau regionaler Entwicklungsunterschiede in Europa ,von unten“ und zur Stärkung lokaler und regionaler Potenziale. In den beteiligten Regionen fördert das gegenseitige Lernen durch Kooperation sowohl das Problembewusstsein für die Entwicklung in Nachbarregionen oder Regionen mit vergleichbaren Rahmenbedingungen als auch für die eigenen Stärken und Schwächen, was die Verbesserung von Lebensbedingungen vor Ort nach sich zieht. Darüber hinaus trägt die transnationale Zusammenarbeit zu einer erfolgreichen Umsetzung von Richtlinien europäischer Fachpolitiken bei. Denn obwohl diese Richtlinien grundsätzlich in nationales Recht überführt werden müssen, ergeben sich staatenübergreifende Herausforderungen, die im Rahmen der transnationalen Zusammenarbeit gelöst werden können. Dies steigert die Erfolgsaussichten und die Effizienz der entsprechenden fachpolitischen Ziele. Über die intensive Einbindung der EU-Mitgliedstaaten und ihrer Regionen ist gewährleistet, dass die konkreten Bedürfnisse vor Ort, in den Regionen und Städten berücksichtigt werden. Die transnationale Zusammenarbeit trägt damit auf verschiedenen Ebenen zur Verwirklichung einer europäischen Integration „,an der Basis“ bei.

\section{Territorialer Zusammenhalt und Raumentwicklung als Gemeinschaftsziele}

Die künftige Berücksichtigung des „territorialen $\mathrm{Zu}$ sammenhalts" als grundlegendem Ziel der Gemeinschaftspolitik unterstreicht die Bedeutung einer integrierten Raumentwicklung. Das Ziel der territorialen Kohäsion, das die wirtschaftliche und soziale Kohäsion ergänzt und erweitert, wird seit seiner Einführung im Vertrag über eine Verfassung für Europa (EVV) intensiv diskutiert. Der Begriff des territorialen Zusammenhalts ist dabei nicht neu; eingeführt wurde er bereits mit Artikel 16 des Amsterdamer Vertrags im Kontext der Dienste von allgemeinem wirtschaftlichem Interesse und taucht in diesem Zusammenhang auch in Artikel 36 der Charta der Grundrechte der Europäischen Union von 2000 auf. Ein konkreter Bezug zum europäischen Territorium und seiner Entwicklung ist dabei jedoch noch nicht erkennbar.

Das EUREK geht in Artikel 29 ebenfalls auf die Förderung des sozialen und territorialen Zusammenhalts als Aufgabe der Gemeinschaft und der Mitgliedstaaten ein. Da sich dieser Artikel auf spezifische EU-Fachpolitiken und deren Einfluss auf die Entwicklung in den europäischen Teilräumen bezieht, wird hier der territoriale Zusammenhalt einerseits in den Kontext einer ausgewogenen und nachhaltigen Entwicklung - als einem der zentralen Ziele der EU - und andererseits einer besseren Koordinierung der raumwirksamen $\mathrm{Ge}$ meinschaftspolitiken gestellt. Insbesondere Letzteres prägt das Verständnis von territorialer Kohäsion in der aktuellen Debatte. Der zweite Kohäsionsbericht widmet dem räumlichen Zusammenhalt ein spezielles Kapitel. ${ }^{11}$ Mit dem dritten Kohäsionsbericht sowie durch seine Berücksichtigung in den EVV als geteilte Kompe- 
tenz zwischen der Europäischen Kommission und den Mitgliedstaaten wird die territoriale Kohäsion endgültig zu einem zentralen Gegenstand der Diskussion zur Raumentwicklung in Europa.

Obwohl es noch keine allgemeingültige Definition des Begriffs gibt, wurde inzwischen deutlicher herausgearbeitet, was territorialer Zusammenhalt umfasst - auch im Unterschied zu integrierter Raumentwicklung. In der alltäglichen Anwendung wird jedoch die Förderung des territorialen Zusammenhalts faktisch gleichgesetzt mit europäischer Raumentwicklung. Dabei versteht die Europäische Kommission den territorialen Aspekt im Rahmen ihrer Kohäsionspolitik fachbereichsübergreifend: Mit der Förderung eines integrierten Konzepts für den territorialen Zusammenhalt will die Kohäsionspolitik mit integrierten Strategien gleichermaßen auf wirtschaftliche, soziale und ökologische Belange eingehen ${ }^{12}$, z.B. bei der Erneuerung, Wiederbelebung und Entwicklung städtischer und ländlicher Gebiete.

Die Mitgliedstaaten der EU haben auf die erweiterten politischen Ziele mit der Erarbeitung der „Territorial Agenda of the European Union" reagiert, die im Rahmen der deutschen Ratspräsidentschaft im Mai 2007 von den zuständigen EU-Ministern verabschiedet wurde. Ziel der Agenda ist es, einen strategischen und umsetzungsorientierten Rahmen für die räumliche Entwicklung in Europa zu liefern und in diesem Sinne durch eine integrierte Raumentwicklungspolitik zur Realisierung der Lissabon- und Göteborgstrategien beizutragen. Mit diesen Zielsetzungen, sowohl vonseiten der Kommission als auch der EU-Mitgliedstaaten, wird eben jener Ansatz in der europäischen Strukturpolitik fest verankert, der der transnationalen Zusammenarbeit von Anfang an immanent war: die Förderung einer integrativen räumlichen Entwicklung in Europa.

\section{Zur Entwicklung der transnationalen Zusammenarbeit seit den 1990er Jahren}

Wie bereits dargestellt, entwickelte sich die transnationale Zusammenarbeit zunächst als Modellprogramm im Rahmen der Gemeinschaftsinitiative INTERREG IIC. Mit dieser Initiative sollten staatenübergreifende Projekte der Raumentwicklung in Europa gefördert werden. Gleichzeitig wurde diese Gemeinschaftsinitiative auch als Umsetzungsinstrument für die Leitvorstellungen des EUREK genutzt.

INTERREG IIC unterschied sich dabei grundsätzlich von den vorangegangenen INTERREG-Initiativen. Mit dem Strang $\mathrm{C}$ wurde die Gemeinschaftsinitiative INTERREG, die sich bis dahin auf die bilaterale grenzüberschreitende Zusammenarbeit konzentrierte, um eine großräumige, staatenübergreifende Zusammenarbeit ausgeweitet. So konnten auch Staaten zusammenarbeiten, die nicht über eine gemeinsame Grenze verfügten. Die Abgrenzung der Kooperationsräume erfolgte im Rahmen politischer Abstimmungen zwischen den beteiligten Staaten und schloss in einigen Fällen auch Nichtmitgliedstaaten der EU mit ein, z. B. im mittel- und südosteuropäischen Raum (CADSES) ${ }^{13}$ oder im Ostseeraum. Die gleichberechtigte Einbeziehung von Drittstaaten in die Entwicklung und Umsetzung von Kooperationsprogrammen ging z.T. über die Maßstäbe der Grenzraumkooperation hinaus und hat nach einer langjährigen widersprüchlichen Entwicklung inzwischen eine neue Qualität innerhalb der europäischen Förderpolitik erreicht. Mit dieser Einbeziehung wurde dem Aspekt Rechnung getragen, dass eine europäische Raumentwicklungspolitik nicht an den Grenzen der EU haltmachen darf, sondern auch die Nachbarstaaten im Sinne einer ausgewogenen und nachhaltigen Entwicklung des europäischen Raums einbinden muss.

Die Zusammenarbeit unter INTERREG IIC erfolgte nach drei Förderschwerpunkten in insgesamt neun transnationalen Kooperationsräumen (sieben für allgemeine transnationale Zusammenarbeit und zwei Programmgebiete zur Verhütung von Überschwemmungen) sowie vier nationalen Programmräumen zur Vorsorge von Dürreschäden. Eine ähnliche Kooperation wurde in vier weiteren Räumen, darunter der Alpenraum, unmittelbar aus den europäischen Strukturfonds gefördert (Pilotaktionen nach Artikel 10 der EFRE-Verordnung). Mit diesen „Pilotmaßnahmen in großen transnationalen Raumordnungsgebieten Europas", die ursprünglich der Vorbereitung der Gemeinschaftsinitiative im Bereich Raumordnung (INTERREG IIC) dienten, sollte „ein Beitrag zur innovativen Weiterentwicklung der Regionalpolitik der Gemeinschaft und der Mitgliedstaaten geleistet werden " ${ }^{14}$. Die Kommission sah in diesen innovativen Maßnahmen einen „ersten Schritt auf dem Weg zu umfassenderen Raumordnungsaktionen und anderen Maßnahmen zur integrierten Verwaltung des Gemeinschaftsraumes, die mehrere Staaten oder Regionen betreffen" ${ }^{15}$

Der finanzielle Schwerpunkt von INTERREG IIC lag im Bereich der Wasserbewirtschaftung, da die Kommission hierin ein typisches raumrelevantes Themenfeld erkannte, bei dem transnationale Zusammenarbeit unstrittig als sinnvoll und notwendig erachtet werden konnte. Für die Programme im Bereich ,vorbeugender Hochwasserschutz" (INTERREG Rhein-Maas-Aktivitäten - IRMA und Frankreich/Italien) und im Bereich „Wasserbewirtschaftung im Zusammenhang mit der Dürrebekämpfung“ (Griechenland, Süditalien, Spanien und Portugal) standen so die meisten Fördermittel 
zur Verfügung. In beiden Bereichen wurden durch die Gemeinschaftsinitiative auch direkt investive Maßnahmen gefördert.

Die Förderung in den übrigen transnationalen Programmen umfasste vorrangig Maßnahmen zur Vorbereitung und Umsetzung transnationaler Strategien, Verbesserung der räumlichen Wirkungen der Gemeinschaftspolitiken, Weiterentwicklung der transnationalen Zusammenarbeit im Bereich der Raumordnung mit Drittstaaten oder Förderung einer nachhaltigen Entwicklung bestimmter Gebietstypen. Im Mittelpunkt standen daher - auch wegen der relativ geringen verfügbaren Mittel - weniger die Förderung investiver Maßnahmen, sondern Planungsaktivitäten, Erfahrungsaustausch, Analysen und Studien. Der Verdienst des INTERREG II C-Programms liegt vor allem im Aufbau von transnationalen Kooperationsstrukturen und dem Austausch zwischen den für die Raumentwicklung zuständigen und relevanten Akteuren.

Die Fortführung der Gemeinschaftsinitiative im Förderzeitraum 2000-2006 war insbesondere auf die positiven Erfahrungen mit INTERREG IIC sowie die Verabschiedung des EUREK zurückzuführen. Das „Experimentierfeld" der transnationalen Zusammenarbeit in der Raumordnung im Rahmen von INTERREG IIC bzw. der innovativen Pilotmaßnahmen nach Artikel 10 EFRE hatte sich bewährt - und es zeigte sich ein großer Bedarf an solchen Kooperationen. So konnten die bereitgestellten Gelder rasch in Projekten gebunden werden, und in vielen transnationalen Räumen wie z.B. im Ostseeraum, Nordwesteuropäischen Metropolraum und CADSES-Raum hätten es angesichts der vorliegenden Projektanträge sogar deutlich mehr Mittel sein können. In diesem Kontext sprachen sich die Europäische Kommission sowie die Mitgliedstaaten und Regionen nachdrücklich für eine Weiterführung der Zusammenarbeit aus. Das EUREK enthält den Ratschlag, die transnationale Zusammenarbeit in der Strukturfondsperiode 2000-2006 im Rahmen von INTERREG III B fortzusetzen und zu intensivieren und gibt hierzu eine Reihe von Empfehlungen. Diese hat die Europäische Kommission in den Leitlinien für die Gemeinschaftsinitiative III aufgegriffen. ${ }^{16}$ Demnach verfolgt INTERREG III das Ziel, durch die Förderung der grenzüberschreitenden, transnationalen und interregionalen Zusammenarbeit zu einer ausgewogenen Entwicklung des Gemeinschaftsraums beizutragen und somit den wirtschaftlichen und sozialen Zusammenhalt in der EU zu stärken. Mit dieser Zielsetzung trägt INTERREG mit seinen drei Handlungssträngen direkt zur Erreichung der zentralen Ziele der EU bei.
Höhere Finanzausstattung, stärker umsetzungsorientiert

Die finanzielle Ausstattung der Gemeinschaftsinitiative wurde deutlich erhöht. Mit 4,9 Mrd. $€$ standen für den Förderzeitraum 2000-2006 rund 2 Mrd. $€$ mehr zur Verfügung als im vorangegangenen Zeitraum. Für die transnationale Zusammenarbeit erhöhte sich das Finanzvolumen von rund 460 Mio. $€$ (einschl. der Pilotaktionen im Alpenraum) auf $1,4 \mathrm{Mrd} . €$ bzw. von 115 auf 186 Mio. $€$ jährlich.

Standen neben den angesprochenen direkten Investitionsmöglichkeiten im Bereich der Wasserbewirtschaftung im Rahmen von INTERREG IIC vor allem der Erfahrungsaustausch, der Aufbau von Akteursnetzen und die Erstellung von Studien und Analysen im Vordergrund, so änderte sich dies in INTERREG III B: Mit der neuen Gemeinschaftsinitiative rückten die Umsetzungsorientierung und kleinere Investitionen stärker in den Fokus der Projektarbeit, wobei die Ergebnisse der vorangegangenen Förderperiode als Grundlage dienten. Trotz der investiven Möglichkeiten war INTERREG III B aber nicht als Investitionsprogramm konzipiert. Der tatsächliche Anteil an Investitionen unterschied sich dementsprechend in den einzelnen Kooperationsräumen deutlich. ${ }^{17}$ Generell lässt sich aber festhalten, dass die Projekte unter INTERREG III B konkreter und stärker aktionsbezogen ausgerichtet waren als noch unter INTERREG II C; reine Forschungs- und Studienprojekte sind in der Minderheit. Gegen Ende der Programmumsetzung wurden zudem Projekte mit stärker strategischer Ausrichtung genehmigt.

\section{Ergebnisse der transnationalen Zusammenarbeit 2000-2006 und künftige Erfordernisse}

In der Förderperiode 2000-2006 wurden in zehn staatenübergreifenden Kooperationsräumen der EU und ihrer Nachbarstaaten sowie in drei Überseeterritorien Raumentwicklungsprojekte umgesetzt (s. Karte 1). Damit sind Netzwerke mit Tausenden von unmittelbaren Projektpartnern und Zehntausenden von Akteuren entstanden. In den Kooperationsräumen, an denen Deutschland beteiligt ist, wirken über 6500 Partner, darunter nahezu 1000 deutsche Partner in rund 500 Projekten zusammen. In den einzelnen Räumen wurden dabei unterschiedliche Erfahrungen hinsichtlich Größe der Projekte, Partnerstruktur, Finanzvolumen, Staatenbeteiligung, Laufzeit u. a. gewonnen, die bei der weiteren Ausgestaltung der Zusammenarbeit ausgetauscht und berücksichtigt werden können. 
Karte 1

INTERREG III B (2000-2006): Co-operation areas

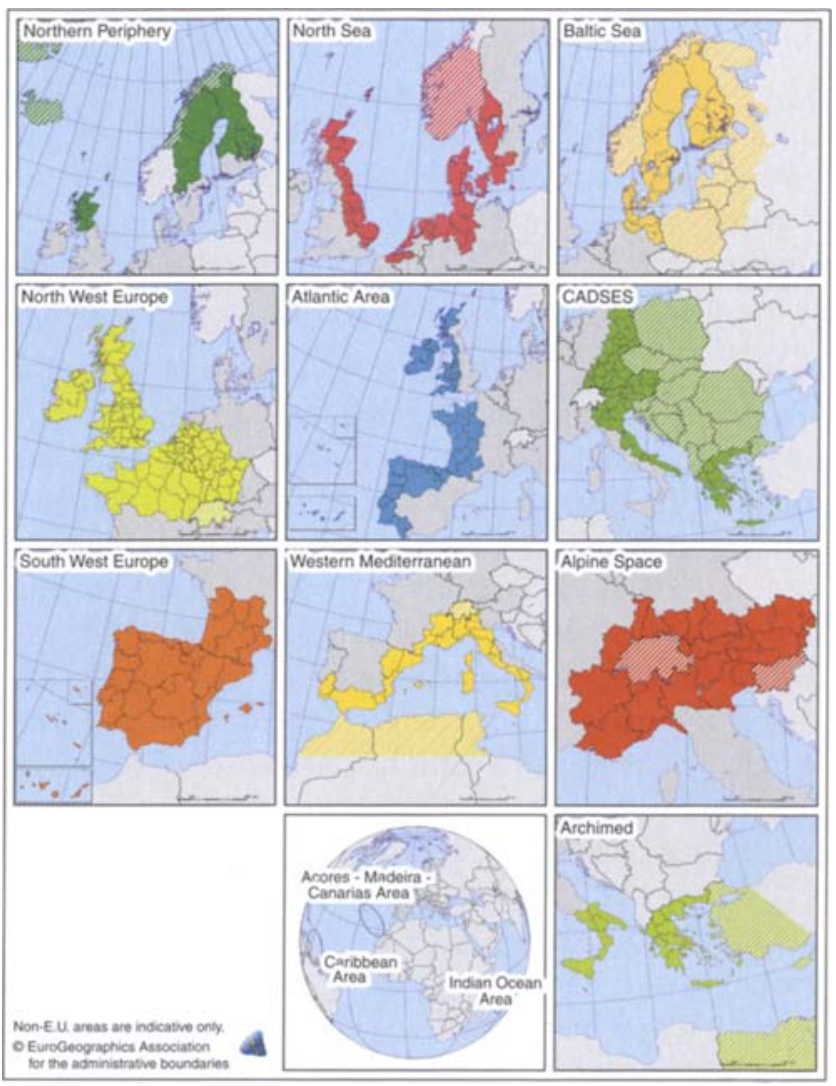

Beispielsweise verfügt der nordwesteuropäische Raum über die umfangreichsten Erfahrungen bei der Förderung finanzstarker Projekte mit größeren Investitionsvorhaben. Im Nordseeraum wurde ein System entwickelt, die Zahl der direkten Projektpartner im Interesse eines effizienten Projektmanagement zu begrenzen, über diese aber weitere Institutionen und Gebietskörperschaften mitwirken zu lassen. Im Ostseeraum wurde eine direkte Unterstützung der Projekte durch Partner besonders vieler Staaten erreicht. (s. Tab. 1).

\section{Städte, Grenzregionen und Staatengruppen als Aktivposten der Kooperation}

In der räumlichen Struktur der Kooperationen treten Großstädte stark hervor. Als Standort vielfältiger hochrangiger Einrichtungen und potenzieller Projektpartner sind sie besonders intensiv in transnationale Projekte eingebunden. Diese Einrichtungen, z. B. Universitäten oder Regierungsbehörden, übernehmen allerdings nicht nur Funktionen für ihren jeweiligen Standort, sondern für größere Räume darüber hinaus. Ein weiteres räumliches Strukturmerkmal der transnationalen Zusammenarbeit ist die Tatsache, dass grenznahe Regionen und Küstenregionen intensiver mitwirken als zentralere, grenzfernere Gebiete der jeweiligen Staaten (s. Karte 2) ${ }^{18}$. Abweichend von solchen Regelmäßigkeiten treten jedoch auch einzelne Städte und Regionen hervor, die relativ unabhängig von Lage und Größe und offenbar eher politisch motiviert besonders intensiv an der transnationalen Kooperation mitwirken. Solche „Aktivposten“ der transnationalen Zusammenarbeit sind neben Metropolen und Hauptstädten gerade auch Groß- und Mittelstädte wie Aalborg, Ancona, Bozen, Danzig, Göteborg, Klaipeda, Lille, Rostock, Tartu und Venedig.

Häufig erweisen sich staatliche Nachbarschaftsbeziehungen als „Motoren“ für die Entwicklung und Umsetzung transnationaler Projekte. So bilden benachbarte Staaten wie etwa Deutschland, Österreich und Italien Finnland und Schweden oder Belgien und die Niederlande besonders häufige Kooperationscluster transnationaler Projekte. ${ }^{19}$

\begin{tabular}{|l|c|c|c|c|c|}
\hline $\begin{array}{l}\text { Programmraum } \\
\text { (Staaten) }\end{array}$ & $\begin{array}{c}\text { Projekte } \\
\text { (Anzahl) }\end{array}$ & $\begin{array}{c}\text { Partner } \\
\text { je Projekt }\end{array}$ & $\begin{array}{c}\text { Staaten } \\
\text { je Projekt }\end{array}$ & $\begin{array}{c}\text { EFRE-Mittel } \\
\text { je Projekt } \\
\text { (Mio. } € \text { ) }\end{array}$ & $\begin{array}{c}\text { Laufzeit } \\
\text { je Projekt } \\
\text { (Monate) }\end{array}$ \\
\hline Alpen (7) & 58 & 11 & 4,7 & 0,9 & 33 \\
CADSES (18) & 134 & 12 & 5,5 & 1,0 & 30 \\
Nordsee (7) & 70 & 8 & 4,8 & 1,8 & 36 \\
Nordwesteuropa (8) & 99 & 9 & 3,7 & 3,3 & 54 \\
Ostsee (11) & 120 & 24 & 6,6 & 1,1 & 30 \\
Insgesamt & 481 & 13 & $\mathbf{5 , 1}$ & $\mathbf{1 , 6}$ & 37 \\
\hline
\end{tabular}

Tabelle 1

Eckdaten der Projekte in transnationalen Kooperationsräumen mit deutscher Beteiligung

Quelle:

INTERREG III B-Projektdatenbank des BBR, Stand 12/2006 
Karte 2

INTERREG III B-Projektorte in den Kooperationsräumen mit deutscher Beteiligung

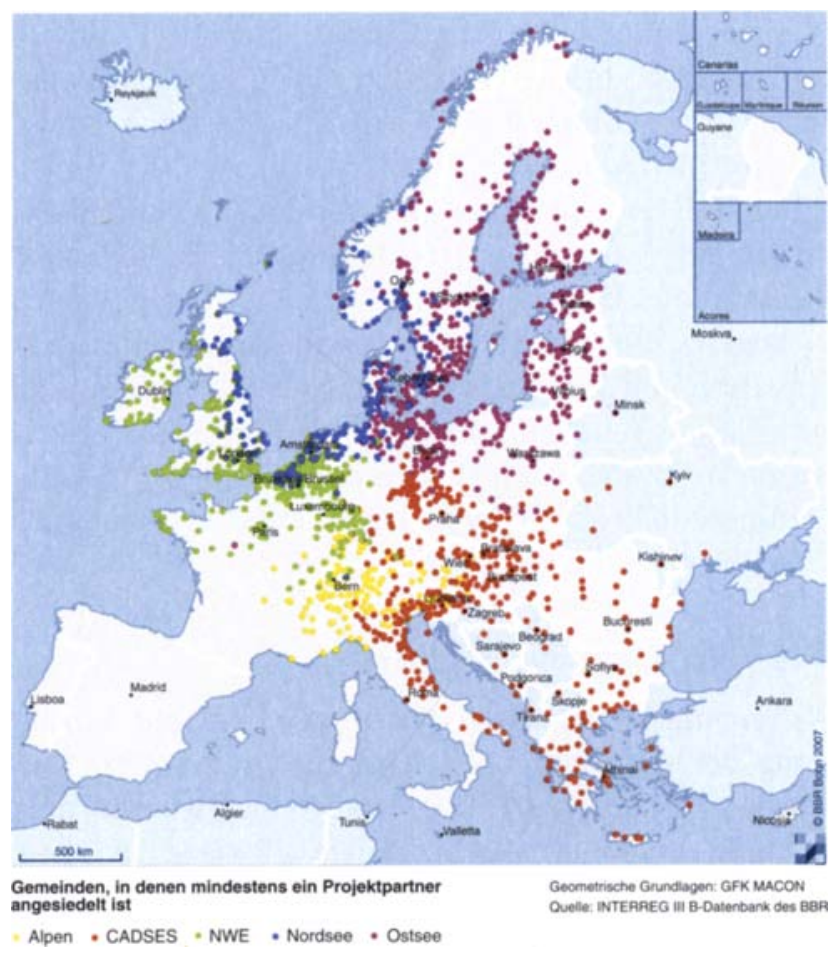

\section{Innovative Projektergebnisse}

Die Projektergebnisse sind vielgestaltig und reichen von transnationalen Studien, Handbüchern und Entwicklungskonzepten bis zu Durchführbarkeitsuntersuchungen, Investitionsstrategien, Planungen und Pilotinvestitionen. Neben Beispiellösungen zu Entwicklungsproblemen bestimmter Raumkategorien (z.B. Entwicklungsperspektiven für ländliche Räume, Flächenmanagement in Stadt-Umland-Regionen, Rüstungskonversionsgebiete) werden Konzepte, Strategien und Aktionen für konkrete Verkehrskorridore, Entwicklungszonen, Flussgebiete sowie transnationale Kulturund Tourismusrouten in staatenübergreifender Kooperation erarbeitet.

Beispielsweise widmet sich das Projekt COMMIN (Promoting Spatial Development by Creating Common Mindscapes) dem Wissenstransfer und der gemeinsamen Qualifizierung im Bereich der Raumplanung und -entwicklung im Ostseeraum. Damit wird bei den sehr unterschiedlichen baltischen, deutschen, nordischen, polnischen, russischen und weißrussischen Planungsund Verwaltungsstrukturen eine Grundvoraussetzung geschaffen, um überhaupt dauerhaft kooperieren zu können. Die gemeinsame transnationale Zusammenarbeit erfordert Mindestkenntnisse der Begriffsinhalte,
Verfahren und Instrumente der internationalen Partner und bietet gleichzeitig die Chance, sich an den fortgeschrittensten Erfahrungen zu orientieren. Das Projekt zielt darauf ab, zukünftige transnationale Projekte und Investitionen im Ostseeraum durch die Entwicklung des Planungsverständnisses und den Aufbau einer gemeinsamen sprachlichen Basis zu unterstützen und eine gemeinsame englischsprachige Terminologie zu entwickeln, womit es auch ausdrücklich die Sprachkompetenz im Ostseeraum fördert. Planer rund um die Ostsee, die sich mit transnationaler Entwicklung beschäftigen, können im Rahmen von COMMIN an Qualifizierungsmaßnahmen teilnehmen, die ihnen einen Zugang $\mathrm{zu}$ fortgeschrittenen Planungssystemen und -ansätzen anderer Ostseeländer sowie Best-PracticeBeispielen aus anderen INTERREG-Projekten bieten. Dabei werden durch ein E-Portal und Informationssystem in allen "Ostseesprachen" sowie Englisch eine gemeinsame Wissensbasis und über Trainingsseminare ein dauerhaftes Weiterbildungsnetzwerk für Planer geschaffen. ${ }^{20}$

Angesichts zunehmender Nutzungskonflikte innerhalb verschiedener wirtschaftlicher Nutzungen und mit der Sicherung natürlicher Lebensgrundlagen haben die Projekte Wadden Sea Forum und Baltcoast Konzepte der Sicherung einer integrierten räumlichen Entwicklung weitergeführt und auch für das "offene Meer“ angewandt. Dabei erwies sich die Verknüpfung traditioneller Konzepte des integrierten Küstenzonenmanagements (IKZM) mit der Raumplanung als vorteilhaft. ${ }^{21}$

Im Bereich des vorbeugenden Hochwasserschutzes und des transnationalen Flussraummanagements sind die Projekte ELLA (Elbe - Labe Flood Management Measures by Transnational Spatial Planning) und ODERREGIO im mittel- und südosteuropäischen Kooperationsraum angesiedelt. Mit den Projekten soll u.a. verhindert werden, dass einseitige Maßnahmen einzelner Staaten oder Regionen z.B. des Hochwassermanagements, der Flussbegradigung, -vertiefung oder -bebauung zu unerwünschten Folgen für Nachbarstaaten und -regionen an den entsprechenden Flussläufen führen. Beispielsweise wird im Projekt ELLA von den elbangrenzenden Ländern und Regionen eine gemeinsame Strategie zum vorsorgenden Hochwasserschutz entwickelt und abgestimmt. Neben dem Austausch zu Erfahrungen und Strategien steht auch eine Institutionalisierung der Zusammenarbeit im Vordergrund. ${ }^{22}$ Eines der Hauptziele von ODERREGIO ist die Erarbeitung eines transnational abgestimmten Handlungsprogramms zum vorsorgenden raumordnerischen Hoch- 
wasserschutz für das Einzugsgebiet der Oder, das die Hochwasserschutzaktivitäten mit Raumbezug im Einzugsgebiet der Oder mittelfristig (bis 2015) leiten soll. ${ }^{23}$

Die Optimierung der vorhandenen Verkehrsinfrastruktur auch zur Umweltentlastung des Alpenraums ist Ziel des Projekts „AlpFRail" (Alpine Freight Railway). Die Verlagerung von Güterverkehr von der Straße auf die Schiene erfolgt in erster Linie durch die Beseitigung von Kapazitätsengpässen und eine Mobilisierung von Potenzialen der Schiene. Dazu wird die gesamte logistische Kette der Warenströme vom Start- zum Zielpunkt quer durch Europa betrachtet, um gemeinsam mit den nationalen und regionalen Akteuren innovative Lösungen zur Schienenlogistik zu entwickeln. ${ }^{24}$

Das Projekt InterPorts (Interports Promotion Net) hat Lösungen zur Verlagerung von Straßen- auf Wasserstraßenverkehr bzw. eine gesteigerte Nutzung von Schienenverkehr im Hinterland von Häfen entwickelt. Binnen- und Seehäfen kooperieren, um gemeinsam den Verkehrsträger Binnenschiff im Seehafen-Hinterlandverkehr sowie das Seeschiff im Kurzstreckenseeverkehr (Short Sea Shipping) zu stärken. Gemeinsame Marktauftritte und verstärkte Kooperationen mit anderen Binnen-, aber auch Seehäfen im In- und Ausland sollen dies ermöglichen. Eine internetbasierte Informationsplattform verbessert die Kooperationsmöglichkeiten der Häfen und kann außerdem von potenziellen Kunden genutzt werden. Im Ergebnis ziehen die einzelnen Binnenhäfen erheblichen Nutzen aus der Vernetzung, da den Kunden abgestimmte Angebote und Alternativen unterbreitet werden können. ${ }^{25}$

\section{Effekte für die Raumentwicklung}

Raumentwicklungspolitisch profitieren sowohl Ballungsräume als auch eher ländlich geprägte Regionen von der Zusammenarbeit. Die gezielte Förderung von Metropolen und anderen Großstädten im Hinblick auf eine bessere Wahrnehmung ihrer Funktionen im globalen Wettbewerb ist zwar in der Tat ein explizites Ziel der transnationalen Programme, darf aber nicht mit einer generellen Förderung von Großstädten verwechselt werden. So beteiligen sich die in Großstädten lokalisierten Einrichtungen auch an Projekten z.B. der Förderung des ländlichen Raums. Grundsätzlich wird angestrebt, die spezifischen Potenziale von Großstädten und wirtschaftsstarken Regionen im globalen Wettbewerb und als „Motoren“ für die regionale Entwicklung insgesamt auszuprägen. Damit kommen entsprechende Projektergebnisse auch wirtschaftsschwächeren Standorten zugute. Gleichzeitig beschäftigen sich zahlreiche Projekte auch und gerade mit spezifischen Entwicklungsalternativen für ländliche bzw. strukturschwächere Regionen.
Neben die unmittelbaren Projektergebnisse treten mittelbare und langfristige Wirkungen. So fördert die konkrete Projektzusammenarbeit ein gemeinsames Problemverständnis über nationale Sichtweisen hinaus und bewirkt durch die Übernahme fortgeschrittener Erfahrungen anderer Regionen und Staaten Änderungen in national üblichen Planungs- und Arbeitsmethoden und -verfahren, von gesetzlichen und finanziellen Regelungen usw. Die Einbindung von Regionen, Gemeinden und Institutionen in staatenübergreifende Netzwerke macht die Standorte und Einzelakteure bzw. -institutionen attraktiver. Und auch bei Investoren sind innovationsfreudige Standorte mit Europakompetenz begehrter. Zudem ergeben sich durch die Projektzusammenarbeit vielfältige Wirtschaftskontakte auch über die eigentlichen Projektpartner hinaus.

\section{Reserven für eine höhere Effizienz}

Neben diesen positiven Wirkungen, die die Umsetzung der transnationalen Förderprogramme im Zeitraum von 2000 bis 2006 gezeitigt hat, wurden aber auch Reserven für deren höhere Effizienz ermittelt. Unabhängig von der weiteren inhaltlichen Ausgestaltung der transnationalen Zusammenarbeit haben zum Beispiel zahlreiche Untersuchungen belegt, dass die Projekte eine größere Breitenwirkung über die unmittelbar beteiligten Akteure hinaus erreichen müssen. ${ }^{26}$ Dies wirft zum einen die Frage nach stärker übertragbaren Modellösungen, zum anderen nach besserer (Fach-)Öffentlichkeitsarbeit und gezielter Nutzung von Massenmedien durch die Projektakteure auf. Weiterer Handlungsbedarf ergab sich aus den eingeschränkten Möglichkeiten, Akteure aus benachbarten Räumen einzubeziehen, um zum Beispiel transnationale Flusssysteme oder Verkehrskorridore vollständig bearbeiten zu können. Auch die Zusammenarbeit mit benachbarten Staaten außerhalb der EU erwies sich trotz erheblicher Fortschritte weiterhin als kompliziert, da unterschiedliche Förderprogramme mit verschiedenartigen Regeln und Entscheidungsmechanismen kombiniert werden mussten. Hier werden im Rahmen der neuen Förderprogramme weitere Fortschritte erwartet, um eine Projektentwicklung und -umsetzung ,aus einer Hand" zu ermöglichen. Als ein Hemmnis auf dem Wege der Erreichung wirtschaftlich und raumentwicklungspolitisch verwertbarer Ergebnisse erwiesen sich schließlich auch die eingeschränkten Möglichkeiten zur Beteiligung privater Partner und zur Formierung von öffentlich-privaten Partnerschaften. Hier müssen künftig geeignetere Formen der Einbeziehung privater Akteure in die Durchführung transnationaler Projekte gefunden werden. ${ }^{27}$ 


\section{Neue Herausforderungen für die transnationale Zusammenarbeit 2007-2013 als „Mainstreamprogamm“}

Nachdem die für den Förderzeitraum 2000-2006 zur Verfügung stehenden Mittel in den transnationalen Kooperationsräumen weitestgehend gebunden sind und sich die Programme in der Abwicklungsphase befinden, wurde im letzten Jahr mit der Erarbeitung der Operationellen Programme für die neue Förderperiode 2007-2013 begonnen. Die Programmräume, an denen Deutschland künftig mitwirkt, sind in Karte 3 dargestellt.

Mit der Einführung des dritten Strukturfondsziels - der europäischen territorialen Zusammenarbeit - wurde die Struktur der europäischen Förderpolitik erweitert. Die bisher im Rahmen der Gemeinschaftsinitiative INTERREG III geförderte grenzraumbezogene, transnationale und interregionale Zusammenarbeit wurde in ein sog. "Mainstreamprogramm“28 der europäischen Strukturpolitik überführt. In der Konsequenz werden sich die inhaltlichen Ziele der transnationalen Zusammenarbeit verschieben. Die Kooperationsprogramme und -projekte werden sich in Zukunft noch stärker daran messen lassen müssen, ob sie einen Beitrag zu den übergeordneten Zielsetzungen der EU leisten und inwiefern sie zur Umsetzung der Lissabon- und Göteborg-Strategien beitragen. Der Kernansatz transnationaler Kooperation liegt dabei - wie in den vorangegangenen Strukturfondsperioden - in der Förderung einer integrierten territorialen Entwicklung. ${ }^{29}$

Künftige transnationale Projekte stehen vor der Herausforderung, aus einem räumlichen Kontext heraus Antworten auf die neuen Anforderungen zu finden. Im Hinblick auf nachhaltiges Handeln wird es zunehmend wichtiger, unterschiedliche Fachpolitiken in die Projektarbeit einzubeziehen und Raumbezüge bei der Gestaltung von Fachpolitiken zu berücksichtigen. Damit wirken die transnationalen Programme in die gleiche Richtung wie die bereits erwähnte "Territoriale Agenda der EU“. Mit ihren erprobten Instrumenten und inhaltlichen Ausrichtungen bieten sie konkrete Möglichkeiten, die wirtschaftliche und soziale Entwicklung mit der Erhaltung der natürlichen Lebensgrundlagen im Sinne der „Territorialen Agenda“ zu verbinden.

Die neuen transnationalen Programme sehen vier thematische Prioritäten vor, die in Projekten bearbeitet werden sollen.
Karte 3

Ziel 3 - Europäische territoriale Zusammenarbeit: Transnationale Kooperationsräume mit Beteiligung Deutschlands

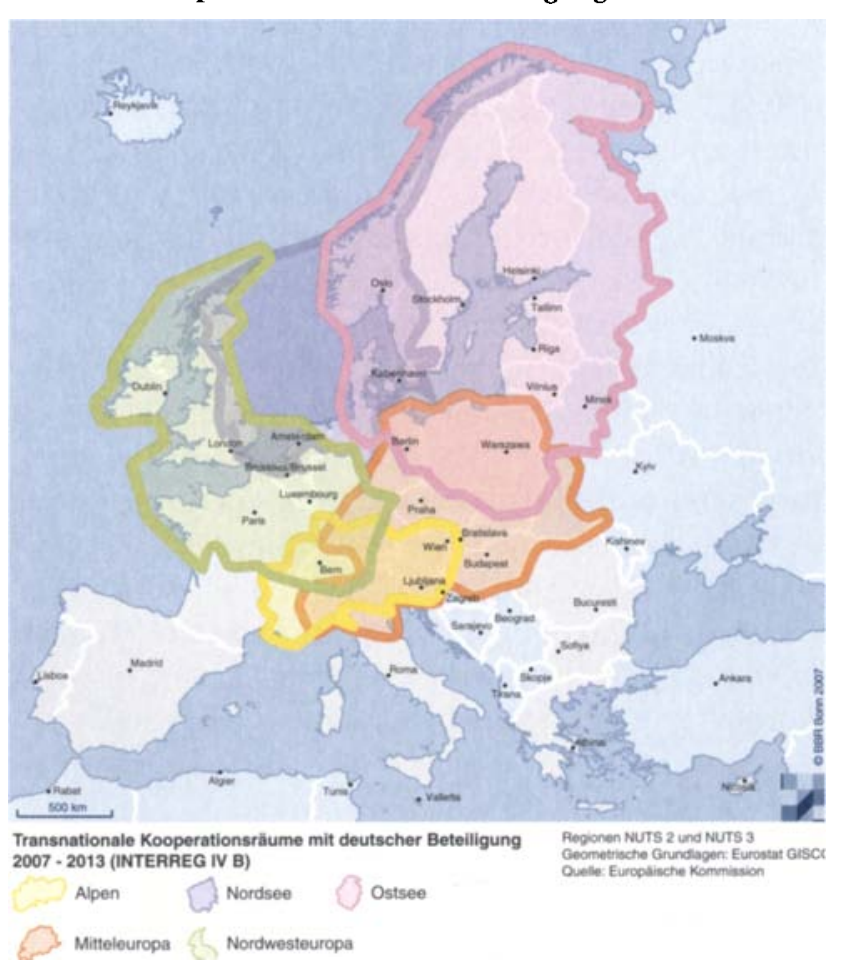

\section{(1) Innovation}

Innovation ist insbesondere vor dem Hintergrund des Ziels, einen Beitrag zur Umsetzung der Lissabon- und Göteborg-Strategien zu leisten, ein wichtiges Thema der künftigen transnationalen Zusammenarbeit. Innovation wird in den neuen Programmen sowohl als eigenständige Priorität für Aktivitäten eingeführt, die insbesondere auf die Verbesserung der organisatorischen, rechtlichen und finanziellen Rahmenbedingungen, des Transfers von Technologie, Wissen und Informationen sowie der Verbreiterung der gesellschaftlichen Basis abzielen. Daneben wird Innovation weiterhin auch horizontal, also alle Förderschwerpunkte betreffend in den Programmen verankert. Da sich die transnationale Zusammenarbeit auf die Förderung der regionalen Entwicklung von Kooperationsräumen konzentriert, stehen nicht die technologische Innovation, sondern die Verbesserung der Rahmenbedingungen und die Förderung einer wissensbasierten Wirtschaft im Vordergrund. Die Vernetzung von Universitäten und Kompetenzzentren sowohl untereinander als auch mit Unternehmen sowie der politischen und administrativen Ebene wird in der transnationalen Zusammenarbeit zukünftig stärkeres Gewicht erhalten. 


\section{(2) Verbesserung des Umweltschutzes und der Risikovorsorge}

Die Verbesserung des Umweltschutzes und der Risikovorsorge, z.B. im Hochwasserschutz an Flüssen und Küsten, ist bereits wesentlicher Bestandteil der Kooperation im Rahmen von INTERREG. Auch in den künftigen Programmen wird dieser Bereich - gerade im Hinblick auf den vorsorgenden Umgang mit dem Klimawandel - eine wichtige Rolle spielen. Schwerpunkte für die Zusammenarbeit werden unter anderem die Herstellung und Umsetzung von Synergien zu EU-Strategien und -Initiativen, die Entwicklung von Konzepten zur maritimen Sicherheit, Beiträge zum verbesserten Schutz der maritimen Umwelt sowie die Entwicklung und Umsetzung transnationaler Strategien zum Umgang mit Risiken und zum Katastrophenschutz sein. Daneben kommt der Förderung des Einsatzes erneuerbarer Energien und der Energieeffizienz in privaten Haushalten und Unternehmen eine wichtige Rolle zu.

\section{(3) Erreichbarkeit}

Beim Thema Erreichbarkeit knüpfen die neuen Programme an die Ergebnisse der laufenden Kooperationen an. Ein stärkerer Fokus liegt allerdings auf der intensiveren Nutzung der vorhandenen Transportinfrastruktur, z.B. durch eine intelligentere Organisation der Verkehre (Multimodalität, Interoperabilität, Nutzung von Kommunikationstechnologien). Im Mittelpunkt zukünftiger transnationaler Zusammenarbeit steht damit nicht die "gebaute" Infrastruktur an sich, sondern vielmehr deren Nutzung und zukunftsfähiges Management vor dem Hintergrund einer nachhaltigen Regionalentwicklung. Große Bedeutung haben weiterhin die positiven Impulse, die Verkehrsinfrastruktur in transnationalen Entwicklungskorridoren setzen kann. Erstmalig ist auch die Förderung des Konzepts der Meeresautobahnen möglich. Weiter relevant sind die Sicherung des Zugangs und der Qualität öffentlicher Dienste und Verkehrssysteme auch in peripheren und dünner besiedelten Regionen.

\section{(4) Nachhaltige Entwicklung der Städte und Regionen}

Eine wichtige Rolle in der künftigen Strukturpolitik nimmt die nachhaltige Entwicklung der Städte und Regionen ein. Dies gilt auch für die territoriale Kooperation. Städte sind "Motoren“ der Regionalentwicklung, und dementsprechend gilt es, städtische Infrastruktur zu stärken und das Management (im Sinn von Governance) zu verbessern. In den neuen Programmen werden Aspekte des demographischen Wandels stärker berücksichtigt als bisher. Auch wird ein stärkeres Gewicht auf die Stadt-Land-Zusammenarbeit gegenüber isolierten Konzepten für den städtischen oder ländlichen Raum gelegt. Das Natur- und Kulturerbe wird weiterhin Bestandteil der Programme sein, hier steht allerdings noch stärker die wirtschaftliche Bedeutung im Vordergrund (Umfeld für Unternehmen, ,weicher“ Standortfaktor).

In den neuen Programmen sollen vermehrt solche strategische Leitprojekte ausgeschrieben werden, die sich durch ihren Einfluss auf den gesamten Kooperationsraum bzw. auf wichtige Teile davon und durch eine starke politische Unterstützung insbesondere der nationalen und internationalen Ebene auszeichnen. Gerade diese Projekte lassen dauerhafte und übertragbare Ergebnisse erwarten, die für die gesamte EU von Relevanz sein können. Dies erfordert eine stärkere politische Kooperation bei der Entwicklung von Projekten und eine intensivere Zusammenarbeit bei der Umsetzung der Programme. ${ }^{30}$ Ansätze dazu bestehen beispielsweise in den umfassenden zwischenstaatlichen Kooperationsstrukturen des Ostseeraums unter dem Dach des Ostseerates. Wichtige Regierungskooperationen sind hier z. B. die Zusammenarbeit der Umweltminister oder der Raumordnungsminister unter der Bezeichnung VASAB $2010^{31}$. Ähnliche Ansätze bestehen bereits im Alpenraum mit der Zusammenarbeit zur Alpenkonvention ${ }^{32}$ oder im Nordseeraum mit der OSPAR-Kommission $^{33}$. In allen Kooperationsräumen hat die Erarbeitung räumlicher Leitbilder die politischstrategische Zusammenarbeit gefördert. ${ }^{34}$

Zukünftige Projekte sollten dazu genutzt werden, eine noch engere Verknüpfung mit anderen Programmen herzustellen, indem zum Beispiel die territorialen Kooperationsprojekte größere Investitionen vorbereiten, die im Rahmen anderer Strukturfondsprogramme, in nationalen Programmen oder mit Hilfe privater Investoren umgesetzt werden. Dazu ist eine stärkere $\mathrm{Zu}$ sammenarbeit verschiedener Verwaltungsebenen in Projekten erforderlich. Bilaterale oder multilaterale Kontakte und Beziehungen nationaler Ministerien können hier Ausgangspunkte für neue Projekte und deren Umsetzung bilden. Künftige Projekte werden sich auch stärker auf Schlüsselthemen der jeweiligen Kooperationsräume konzentrieren, die einer transnationalen Lösung bedürfen. Sektorspezifische Maßnahmen, die nicht zur wirtschaftlichen Entwicklung oder zur allgemeinen räumlichen Entwicklung beitragen, werden ebensowenig gefördert wie Aktivitäten mit ausschließlich lokalem oder regionalem Mehrwert. Darüber hinaus gilt es, die Wirksamkeit und Sichtbarkeit von Projektergebnissen zu erhöhen und eine professionellere Kommunikation darüber zu entwickeln. 
Neben der thematischen Profilierung wird die transnationale Zusammenarbeit künftig noch stärker dabei helfen, das Profil der einzelnen Räume auszuprägen. Die Zusammenarbeit wird dabei im Wesentlichen in den bestehenden Kooperationsräumen fortgeführt. Im Interesse einer wirksameren Programmumsetzung und einer besseren Berücksichtigung spezieller Bedingungen einzelner Räume wurden jedoch ein gemeinsames Mittelmeer-Programm gebildet sowie das Programm für den CADSES-Raum in zwei Einzelprogramme geteilt. In einem bestimmten Umfang werden künftig auch Kooperationsmöglichkeiten über die einzelnen Räume hinaus mit anderen Regionen der EU sowie Nachbarstaaten eröffnet. Dies geschieht vor allem durch Einbindung thematisch geeigneter Akteure.

Insgesamt kann die transnationale Zusammenarbeit künftig noch stärkere Beiträge dazu leisten, sowohl „Europa von unten“ durch konkrete transnationale Kooperationsprojekte zusammenwachsen zu lassen als auch strategische Zukunftsentscheidungen durch staatenübergreifende Aktivitäten vorzubereiten. ${ }^{35}$

\section{Anmerkungen}

(1)

Vgl. Europäische Raumordungsministerkonferenz (CEMAT), Europäische Raumordnungscharta - Torremolinos-Charta, angenommen auf der sechsten Europäischen Raumordnungsministerkonferenz in Torremolinos, Spanien, 1983

(2)

Selke, Welf: Europäische Raumordnungspolitik nach Maastricht. Inform. z. Raumentwicklung (1993) H. 9/10, S. 577-583

(3)

Vgl. Schön, Karl Peter: Das Europäische Raumentwicklungskonzept und die Raumordnung in Deutschland. Einführung. Inform. z. Raumentwicklung (2000) H. 3/4, S. I-VII

(4)

Europäische Gemeinschaften - Kommission: Europa 2000 - Perspektiven der künftigen Raumordnung der Gemeinschaft.

- Luxemburg 1991

(5)

Europäische Kommission: Europa 2000+ - Europäische Zusammenarbeit bei der Raumentwicklung. - Luxemburg 1995

(6)

Bundesministerium für Raumordnung, Bauwesen und Städtebau (Hrsg.): Grundlagen einer Europäischen Raumentwicklungspolitik. - Bonn 1995, S. 29

(7)

Vgl. Ahlke, Brigitte: Transnationale Zusammenarbeit in der Raumordnung: neue Ansätze, Programme, Initiativen. Inform. z. Raumentwicklung (1997) H. 6, S. 361-378
(8)

Europäische Kommission: Europa 2000+, a. a. O.

(9)

Vgl. Europäische Kommission (Hrsg.)/Ausschuss für Raumentwicklung: EUREK - Europäisches Raumentwicklungskonzept. Angenommen beim Informellen Rat der für Raumordnung zuständigen Minister in Potsdam, Mai 1999. - Luxemburg 1999, Einleitung

(10)

Ebda., Abs. 61

(11)

Vgl. Europäische Kommission: „Einheit Europas, Solidarität der Völker, Vielfalt der Regionen“. Zweiter Bericht über den wirtschaftlichen und sozialen Zusammenhalt. - Luxemburg 2001, S. $29 \mathrm{ff}$.

(12)

Vgl. Kommission der Europäischen Gemeinschaften: Vorschlag für eine Entscheidung des Rates über strategische Kohäsionsleitlinien der Gemeinschaft (KOM (2006) 386, S. 6 sowie S. 36 ff.

(13)

CADSES $=$ Central - , Adriatic-, Danubian and South-Eastern European Space (Mitteleuropäischer, Adriatischer, Donau- und Südosteuropäischer Raum)

(14)

Brigitte Ahlke: Transnationale Zusammenarbeit in der Raumordnung, a. a. O., S. 366

(15)

Vgl. Europäische Kommission (Hrsg.): Leitfaden für innovative Maßnahmen zur regionalen Entwicklung (Art. 10 der EFRE-VO) 1995-1999. - Luxemburg 1995, S. 29

(16)

Vgl. hierzu ausführlicher Ahlke, Brigitte: INTERREG II C - Transnationale Programme und Projekte zur Umsetzung des EUREK. Inform. z. Raumentwicklung (2000) H. 3/4, S. 157 ff.

(17)

Vgl. Bundesamt für Bauwesen und Raumordnung (Hrsg.), Transnationale Zusammenarbeit - TransCoop 05 Report. - Bonn 2005. $=$ Berichte, Bd. 22, S. $17 \mathrm{ff}$.

(18)

Die im Beitrag verwendeten Karten wurden mit Ausnahme von Karte 1 von Dirk Gebhardt im BBR erarbeitet.

(19)

Vgl. Görmar, Wilfried: Wer kooperiert mit wem in transnationalen Projekten? Inform. z. Raumentwicklung (2005) H. 11/12, S. 675 ff.

(20)

Weitere Informationen siehe unter www.commin.org

(21)

Vgl. auch www.waddensea-forum.org

(22)

Vgl. www.ella-Interreg.org 
(23)

Vgl. www.oderregio.org

(24)

Vgl. www.alpfrail.com

(25)

Vgl. www.interports.de

(26)

Vgl.: Böhme, K.; Josserand, F.; Haraldsson, P. I.; Bachtler, J.; Polverari, L.: Transnational Nordic-Scottish Co-operation. Lessons for Policy and Practice. - Stockholm 2003

(27)

Vgl.: Bundesamt für Bauwesen und Raumordnung (Hrsg.): Transnationale Zusammenarbeit, a. a. O., S. 112

(28)

Hierzu zählen alle Programme im Rahmen der Hauptziele der Förderpolitik der EU, nunmehr Konvergenz, Wettbewerbsfähigkeit und Beschäftigung sowie territoriale Zusammenarbeit.

(29)

Vgl. Art. 6 Abs. der EFRE-Verordnung (Verordnung (EG) Nr. 1080/ 2006 des Europäischen Parlaments und des Rates vom 5. Juli 2006 über den Europäischen Fonds für regionale Entwicklung

(30)

Vgl:: Görmar, Wilfried: Zur Zusammenarbeit zwischen den Regionen Europas auf dem Gebiet der regionalen Strukturpolitik. In: Grenzüberschreitende Zusammenarbeit zwischen den Regionen in Europa. Hrsg.: Xuewu Gu. - Baden-Baden 2002. = Schriften des Zentrums für Europäische Integrationsforschung (ZEI), Bd.39, S. 51-68 (56 f.)

(31)

Das weltweit erste Leitbild für die räumliche Entwicklung einer großen staatenübergreifenden Region entstand 1994 als Ergebnis der Zusammenarbeit der für Raumordnung und -entwicklung zuständigen Minister der Ostseeanrainer und -nachbarstaaten. Dieses Leitbild "Vision and Strategies around the Baltic Sea 2010" wurde später unter dem Kürzel „VASAB 2010“ namensgebend für diese Kooperation. Es entstand in enger Wechselbeziehung zum EUREK.
(32)

Die Alpenkonvention wurde 1991 von den Umweltministern der Alpenländer Deutschland, Schweiz, Österreich, Italien, Frankreich, Slowenien, Liechtenstein, Monaco sowie der EU verabschiedet und ist seit dem 6.3.1995 in Kraft. Sie beinhaltet auch eine koordinierte Zusammenarbeit in verschiedenen Fachbereichen.

(33)

Die OSPAR-Kommission koordiniert die internationale Zusammenarbeit zum Schutz der marinen Umwelt im Nordost-Atlantik. Sie arbeitet auf der Grundlage der Abkommen von Oslo 1972 und Paris 1974 (www.ospar.org).

(34)

Vgl. hierzu: Vision Planet - Strategien für eine integrierte Raumentwicklung im mitteleuropäischen, Donau- und Adriaraum. Angenommen auf der Sitzung des Projektpanels am 12. Januar 2000 in Wien; Norvision: A spatial perspective for the North Sea region. Vision Working Group with representatives from spatial planning offices from the participating countries and regions prepared by the Planco Consulting GmbH. - Essen 2000; Arbeitsgruppe „Räumliches Leitbild“: Ein Räumliches Leitbild für Nordwesteuropa. Kooperationen schaffen. - Den Haag, September 2000

(35)

Für weitere Informationen siehe www.interreg.de

Brigitte Ahlke

Dr. Wilfried Görmar

Jens Kurnol

Nicole Schäfer

Bundesamt für Bauwesen und Raumordnung

Deichmanns Aue 31-37

53179 Bonn

E-Mail: Brigitte.Ahlke@bbr.bund.de Wilfried.Goermar@bbr.bund.de Jens.Kurnol@bbr.bund.de Nicole.Schaefer@bbr.bund.de 